СТАТИЧЕСКАЯ МОДЕЛЬ ПЬЕЗОЭЛЕКТРИЧЕСКОГО ГИРОСКОПА-АКСЕЛЕРОМЕТРА В РЕЖИМЕ ИЗМЕРЕНИЯ УГЛА ОТКЛОНЕНИЯ

DOI: $10.31618 /$ ESU.2413-9335.2019.1.62.86

Газарханов Энвер Тапдыг оглы

Д.т.н., профессор кафедры «Авионика»

Национальной Академии Авиачии Азербайджана

Каримли Тогрул Иса огль

К.т.н., доиен кафедры «Авионика»

Национальной Академии Авиаџии Азербайджана

Нейматов Васиф Айдын огль

Дочент кафедры «Аэрокосмические приборы»

Нациинальной Академии Авиации Азербайджана

Каримли Васиф Иса огль

К.т.н., капитан ВС «А 319/220», 3АО «Азербайджан Хава Йоллары»

\title{
COMPUTER MODEL OF THE PIEZOELECTRIC GYROSCOPE ACCELEROMETER IN THE MODE OF MEASUREMENT OF THE ANGLE OF THE DEVIATION
}

\author{
Hazarkhanov Anavr Tapdig, \\ Karimli Toghrul Isa, \\ Neymatov Vasif Aydin, \\ Karimli Vasif Isa
}

\section{АННОТАЦИЯ.}

В представленной статье изложены конструктивные особенности и принцип работы пьезоэлектрического гироскопа-акселерометра. Обоснован выбор электрической мостовой схемы соединения пьезоэлектрических чувствительных элементов. Составлены математические уравнения для выходного напряжения мостовой схемы с учетом емкостного характера пьезоэлектрического эффекта и компьютерная модель. На основе результатов симуляции компьютерной модели математических уравнений, составленных для электрической мостовой схемы с идентичными пьезоэлементами сделаны необходимые выводы.

\section{ABSTRACT.}

In the submitted article design features and the principle of work of a piezoelectric gyroscope - the accelerometer in brief is stated. The choice of an electric bridge circuit of connection of piezoelectric detectors is reasonable. Mathematical equations for the voltage output of a bridge circuit taking into account the capacity nature of a piezoelectric effect and computer model are worked out. On the basis of the results of simulation of the computer model of mathematical equations, the necessary conclusions are made for the electric Bridge scheme with identical piezoelectric elements.

Ключевые слова: гироскопы и акселерометры; пьезоэлектрический преобразователь; электрическая схема соединения; статические характеристики; компьютерная модель.

Keywords: gyroscopes and accelerometers; piezoelectric converter; electric bridge circuit; static characteristics; computers model.

Введение. Инерциальная навигационная система (ИНС), состоящая из гироскопов и акселерометров, предназначена для определения координат, углов наклона стационарных и нестационарных объектов, а также направления движения и угловых скоростей различных подвижных элементов управления, подводных и надводных кораблей, летательных аппаратов [1].

В Национальной Авиационной Академии Азербайджанской Республики ведутся научно-исследовательские работы по созданию новых пьезоэлектрических гироскопов-акселерометров (ГА) с более повышенной чувствительностью и с пониженными массо-габаритными показателями. В частности, на рис. 1 приведена конструктивная схема ГА. Представленное конструктивное исполнение было предложено на основе критического анализа ГА [2-7]. Характерными недостатками этих ГА являются: излишняя аппаратная избыточность, дополнительная затрата времени для точной перпендикулярной настроек осей чувствительности в перпендикулярной плоскости, низкая чувствительность, большая погрешность измерения, необходимость введения исходных угловых данных по сигналам акселерометров и т.п.

В работах $[8,9]$ исходя из анализа обобщенных характерных недостатков ГА современных ИНС сформированы основные задачи их усовершенствования с возможностью параллельного измерения различных параметров (линейное и угловое ускорение, угловая скорость, угловое положение), увеличения диапазона измерений линейного ускорения, а также коррекции (настройки) измеряемых углов наклона путем использования силы гравитации.

Специфика технического решения данной задачи в представленном варианте конструктивного исполнения разработанного пьезоэлектрического самонастраивающегося гироскопа-акселерометра (ПСГА) (рис.1) заключается в том, что чувствительные к ускорению элементы, расположенные на самых крайних противоположных точках подвижного объекта и фиксирующие величины линейного 
и углового ускорений, а также угловой скорости, при его отклонении относительно вертикальной плоскости подвергаются максимальному перемещению, преодолевая максимальный дифференциальный отрезок. При этом инерционные массы, подвергаясь максимальному перемещению, своей упругой прокладкой с наибольшей силой воздействуют на чувствительные пьезоэлементы.

Основным преимуществом пьезоэлектрического самонастраивающегося ПСГА можно считать:

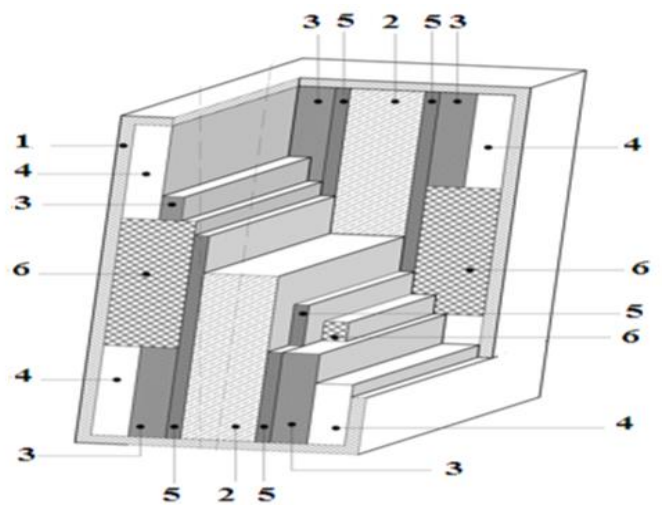

Рис. 1. Пьезоэлектрический гироскоп-акселерометр

1-корпус; 2-возбудительный пьезоэлемент; 3-чувствительные пьезоэлементы;

4-инериионные массы; 5-упругие прокладки; 6-ограничитель

При перемещении оси чувствительности из положения абсолютного вертикального положения, например вправо под воздействием линейного и (или) углового ускорений, угловой скорости перемещения инерционных масс 3 с правой стороны (верхней и нижней части), воспринимаемые упругими прокладками 4, способствуют увеличению прижимного усилия на правосторонних чувствительных пьезоэлементах, в то же время перемещения левосторонних инерционных масс (верхней и нижней части), воспринимаемые упругими прокладками, способствуют уменьшению прижимного усилия на левосторонних чувствительных пьезоэлементах.

Расположение инерционных масс, прокладок, чувствительных пьезоэлементов на концевых точ-
- возможность одновременного измерения линейного и углового ускорений, а также угловой скорости, что значительно повышает эффективность измерений, причем отсутствие частоты ограничения позволяет увеличить диапазон измерений линейного ускорения;

- измеренные углы наклона непрерывно автономно корректируются (настраиваются). ках возбудительного пьезоэлемента (драйвера) способствуют наибольшему линейному перемещению этих точек при поворотном движении устройства.

Постановка задачи. Очевидно, что пьезоэлектрические чувствительные элементы (ПЧЭ) 2 данного ПСГА должны быть соединены по мостовой схеме (рис. 2).

Как известно, выходное напряжение электрического моста определяется формулой $[10,11]$ :

$$
U=\frac{Z_{1} Z_{4}-Z_{2} Z_{3}}{\left(Z_{1}+Z_{2}\right)\left(Z_{3}+Z_{4}\right)} E .
$$

где $E$-электродвижущая сила источника питания; $Z_{1}, Z_{2}, Z_{3} Z_{4}$-комплексные сопротивления плеч электрического моста (в данном случае ПЧЭ).

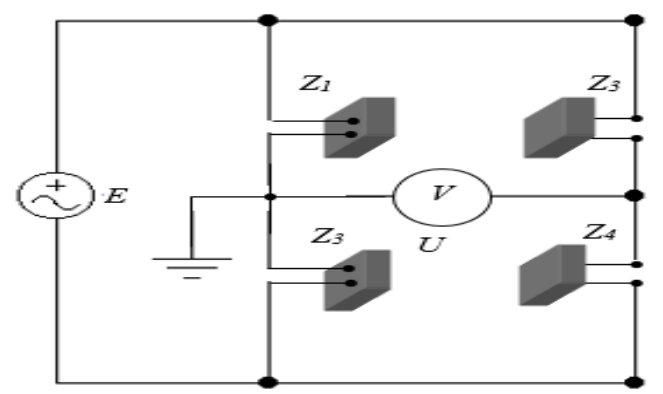

Рис.2. Электрическая схема соединения пьезоэлектрических чувствительных элементов

Если принять во внимание тот факт, что все пьезоэлектрические чувствительные элементы устройства идентичны между собой по всем параметрам, то в уравновешенном состоянии:

$$
Z_{1}=Z_{2}=Z_{3}=Z_{4}=Z_{0}
$$

$Z_{0}$ - комплексное сопротивление ПЧЭ соответствующее резонансной частоте.
Можно подключить ПЧЭ к плечам моста таким образом, чтобы при работе устройства сопротивления моста изменились следующим образом:

$$
\begin{aligned}
& Z_{1}=Z_{4}=Z_{0}+\Delta Z ; \\
& Z_{2}=Z_{3}=Z_{0}-\Delta Z
\end{aligned}
$$

Тогда выходное напряжение, выражаемое (2): 


$$
U=\frac{\left(Z_{0}+\Delta Z\right)^{2}-\left(Z_{0}-\Delta Z\right)^{2}}{\left(\left(Z_{0}+\Delta Z\right)+\left(Z_{0}-\Delta Z\right)\right)^{2}} E
$$

а после несложных математических преобразований может быть заменено в виде:

$$
U=\frac{E}{Z_{0}} \Delta Z
$$

Таким образом, с целью исследования статических характеристик с помощью составленной компьютерной модели. необходимо на основе выражения (4) составить уравнение выходного напряжения ПСГА в зависимости от измеряемого угла отклонения

Решение задачи. Согласно принципу работы гироскопа-акселерометра, в зависимости от его углового положения, происходит отклонения значения частоты от резонансного. То есть, если комплексное сопротивление электрической эквивалентной схемы (ЭЭС) ПЧЭ, соответствующее к резонансной частоте определится как:

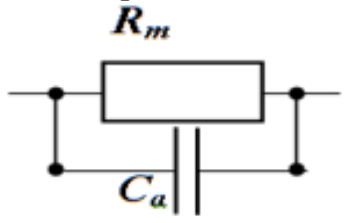

a)

$$
Z_{0}=\frac{R_{m} Z_{c a}}{R_{m}+Z_{c a}}
$$

а в общей форме, как:

$$
Z_{a}=\frac{Z_{m} Z_{c a}}{Z_{m}+Z_{c a}}
$$

то отклонение от резонансной частоты даст разность сопротивлений:

$$
\Delta Z=Z_{a}-Z_{0}=\frac{Z_{m} Z_{c a}}{Z_{m}+Z_{c a}}-\frac{R_{m} Z_{c a}}{R_{m}+Z_{c a}} \text {,(7) }
$$

или же:

$$
\Delta Z=\frac{Z^{2} c a\left(Z_{m}-R_{m}\right)}{\left(Z_{m}+Z_{c a}\right)\left(R_{m}+Z_{c a}\right)}
$$

Где $R_{m}$-активное сопротивление, $Z_{m}$ - комплексное сопротивление динамической части; $Z_{c a}$ -комплексное сопротивление параллельной части ЭЭС ПЧЭ (рис.3)

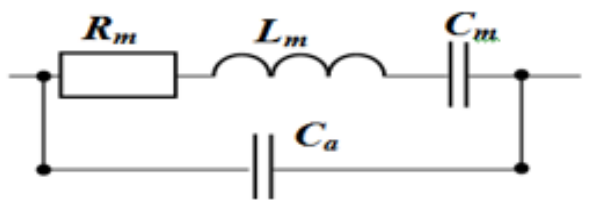

б)

Рис.3. Электрическая эквивалентная схема ПЧЭ а) при резонансе; б) в общей форме

С учетом выражений (5), (8) в (4) и после преобразований, получится:

$$
U=\frac{Z_{c a}\left(Z_{m}-R_{m}\right)}{\left(Z_{m}+Z_{c a}\right) R_{m}} E
$$

Как видно из выражения (9), при резонансе: $Z_{m}=R_{m}$, поэтому $U=0$.

Если принять условие, согласно которому пьезоэлектрическое преобразование имеет только емкостной эффект, то тогда в выражении для комплексного сопротивления динамической части ЭЭС ПП, составленное согласно схеме, приведенной на рис. 3б:

$$
Z_{m}=\sqrt{R_{m}^{2}+\left(\omega L_{m}-\frac{1}{\omega C_{m}}\right)^{2}}
$$

нужно записать формулу для емкости:

$$
C_{m}=\frac{\varepsilon \varepsilon_{0} b l}{a}
$$

а также учесть тот факт, что согласно принципу работы устройства, при воздействии силы на ПЧЭ меняется толщина $a$ под действием упругой силы, создаваемой силой тяжести инерционной массы, выражение (10) можно написать в виде:

$$
Z_{m}=\sqrt{R_{m}^{2}+\left(\omega L_{m}-\frac{a+\frac{m g \sin (\alpha)}{k_{p}}}{\omega \varepsilon \varepsilon_{0} b l}\right)^{2}}
$$

Где $a, b, l$ - толщина, ширина и длина ПЧЭ; $m$-масса инерциального элемента (см: рис.1, поз.3); $\kappa_{p}-$ коэффициент упругости ПЧЭ; $\omega$ - угловая частота напряжения питания; $\alpha$ - угловое отклонение, измеряемое ПСГА; $\varepsilon$-относительная диэлектрическая проницаемость ПЧЭ; $\varepsilon_{0}-$ электрическая постоянная; g- ускорение свободного падения.

Таким образом, выражение для выходного напряжения ПСГА в зависимости от угла отклонения будет иметь вид: 


$$
U=\frac{\left(\sqrt{R_{m}^{2}+\left(\frac{\omega^{2} L_{m} k_{p} \varepsilon \varepsilon_{0} b l-k_{p} a-m g \sin (\alpha)}{k_{p} \omega \varepsilon \varepsilon_{0} b l}\right)^{2}}-R_{m}\right)}{\left.\left(\omega C_{a} \sqrt{R_{m}^{2}+\left(\frac{\omega^{2} L_{m} k_{p} \varepsilon \varepsilon_{0} b l-k_{p} a-m g \sin (\alpha)}{k_{p} \omega \varepsilon \varepsilon_{0} b l}\right)^{2}}+1\right) R_{m}\right)} E
$$

На рис. 4. показана компьютерная статическая модель пьезоэлектрического преобразователя, составленная на основе выражения (13), а также нижеприведенных уравнений, которые представлены в литератype [12]:

$$
\begin{array}{r}
L_{m}=\rho \frac{a l}{8 b} \frac{\left(s_{33}^{E}\right)^{2}}{8 d_{13}^{2}} \\
C_{m}=\frac{8 b l}{h^{2} \pi^{2} a} \frac{d_{13}^{2}}{8 s_{33}^{E}} \\
R_{m}=\frac{8 h^{2} \pi^{2}}{8 b l} \frac{8\left(s_{33}^{E}\right)^{2}}{d_{13}^{2}} \\
C_{0}=\frac{e_{11}^{S} b l}{a} \\
\omega=2 \rho i f=\frac{1}{\sqrt{L_{m} C_{m}}}
\end{array}
$$

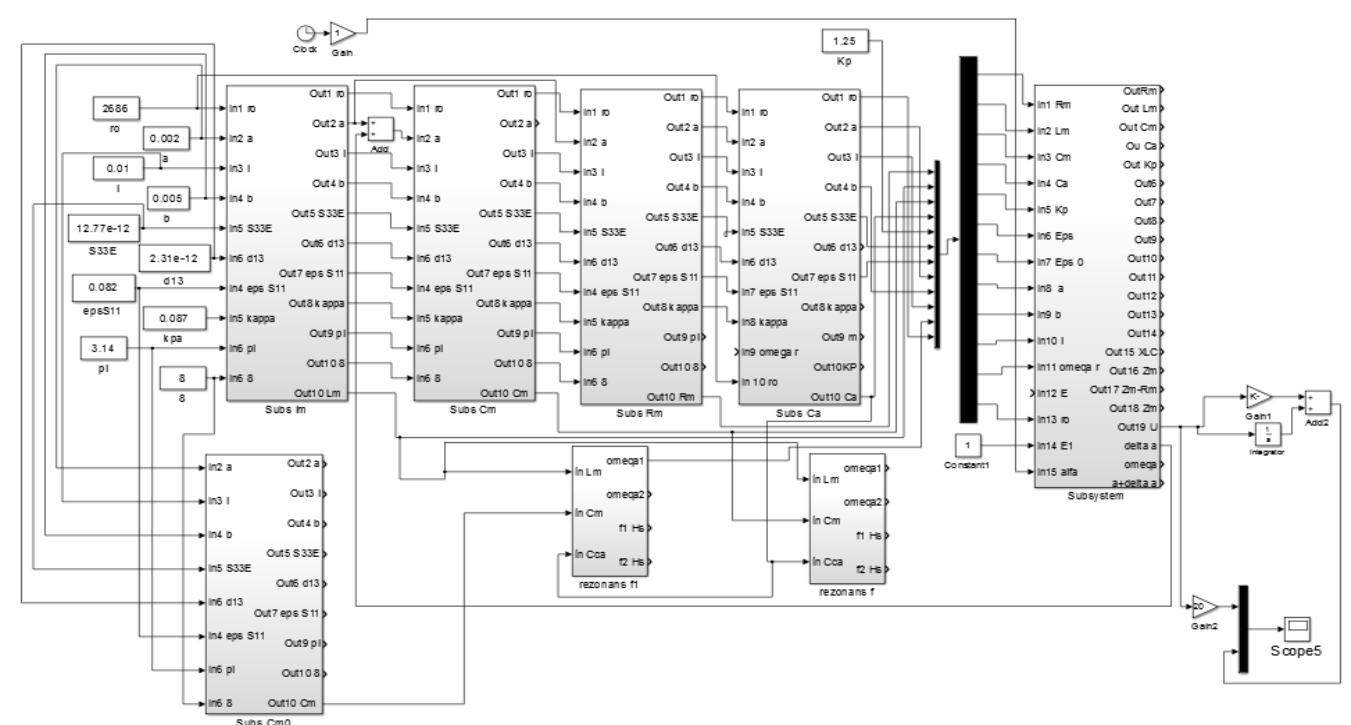

Рис.4. Статическая модель пьезоэлектрического преобразователя

Блоки Subs Lm, Subs Cm, Subs Rm, Subs Ca coставлены на основе уравнений (14-17) соответственно, блок rezonans f на основе уравнений (18), и блок Subsystem на основе уравнения (13). Данные для расчета взяты из литератур $[13,14]: h=1 ; \rho$ $=2686 \kappa 2 / \mathrm{s}^{3} ; a=0,002 \mu ; l=0,01 \mu ; b=0,005 \mu ; s_{33}^{E}$
$=12,77 ; d_{13}=2,31 \mathrm{e}-12 ; e_{11}^{S}=2,31 \mathrm{e}-12 ; E=24 B$. Получены численные результаты: $L_{m}=41,14$ Гн; $C_{m}=$ $8,476 \mathrm{e}-15 \Phi ; R_{m}=131,1 O_{M} ; f_{p}=26980$ Гu ; $\alpha=0-90^{0}$; $U=0-0.18 B$.

Статическая характеристика, показывающая зависимость выходного напряжения от угла отклонения при разных частотах приведены на рис.5. 


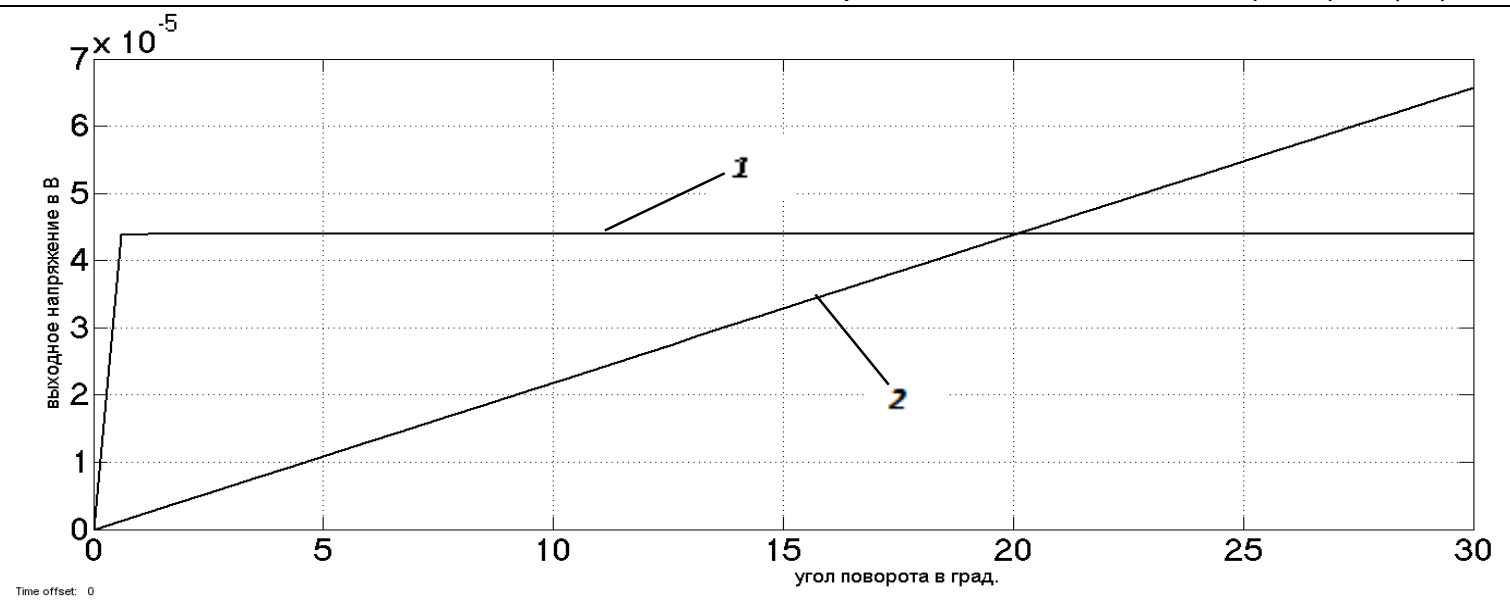

Рис.5. Осииллограммы, полученные от симуляции компьютерной модели пьезоэлектрического преобразователя.

1-график изменения выходного напряжения без корректирующего устройства; 2- с корректирующим устройством.

Как видно из рис.5, выходное напряжение пьезоэлектрического преобразователя при малых градусах угла поворота имеет острый линейно-возрастающий характер, а при больших градусах, скорость изменения выходного напряжения, сильно уменьшается и по существу является нелинейным.

Заключение. При подключении ПЧЭ разработанного ПСГА, имеющий пьезоэлектрический эффект с емкостным характером по электрической мостовой схеме соединения, скорость изменения выходного напряжения намного больше при измерении малых углов поворота, чем в остальной части диапазона измерения угла поворота.

$\mathrm{C}$ целью расширения диапазона измерений с линейным выходным напряжением, необходимо принять дополнительные меры. В частности, можно применить пропорционально-интегрирующее корректирующее устройство: $K u+(1 / s)$ Подбором значений для пропорционального коэффициента $K u$ было установлено, что для обеспечения изменения выходного напряжения пьезоэлектрического гироприбора по приблизительно- линейному закону по всему диапазону измерения угла поворота, при приведенных вышеуказанных значений параметров, должен удовлетворить условие $K u \leq 0,05$.

\section{Библиографический список}

1.David L. Churchill. Inertial measurement system with self correction: Patent US 8,010,308 B1 Int. Cl.: G01C 25/00 (2006.01), G06F 19/00 (2011.01). Aug. 30, 2011, p.20.

2. Компания "ST Software" [Электронный peсурс]. - 2018. - Режим доступа: https://www.stsoftware.nl/index.html.

3. Распопов В.Я. Микромеханические приборы: Учебное пособие. М.: Машиностроение, 2007, 400c.

4. Матвеев В.В. Математические модели микромеханических гироскопов LL-типа//Известия ТулГУ. Технические науки.2012. Вып.12, Ч1, с.205213.
5. Матвеев В.В., Погорелов М.Г. Анализ погрешностей микромеханических гироскопов методом вариаций Аллана//Известия ТулГУ. Технические науки, 2015, Вып.3, с.123-135.

6. Петров П.Н. Акустика. Электроакустические преобразователи: Учебное пособие. СПб.: СПбГУАП, 2003, 80c.

7. Анцев Г.В., Богословский С. В., Сапожников Г. А. Гироскоп-акселерометр. Patent: RU 2381510, 10.02.2010, 9c.

8.Каримли Т.И., Каримли В.И. Пьезоэлектрический самонастраивающийся гироскоп акселерометр. Описание изобретения к евразийской заявке: EA 201500859 A1. 31.10.2016. 12c

9.Пашаев А.М., Каримли Т.И., Нейматов В.А. Исследование схемы измерительной цепи с пьезоэлектрическим дифференциальным акселерометром // Вестник Азербайджанской Инженерной Академии. Том 9, №2. Баку, 2017, с.7-14.

10. Шабалдин Е.Д. Метрология и электрические измерения: Учеб. пособие / Шабалдин Е.Д., Смолин Г.К., Уткин В.И., Зарубин А.П.; Под ред. Е.Д. Шабалдина. Екатеринбург: Изд-во ГОУ ВПО «Рос. гос. проф.- пед. ун-т», 2006. 282 с.

11. Малиновский В.Н., Демидова-Панферова Р.М..Электрические измерения. Учебное пособие для вузов. /под ред. Малиновского В.Н. М: Энергоатомиздат, $1985.416 \mathrm{c}$.

12. Зеленка И. Пьезоэлектрические резонаторы на объемных и поверхностных акустических волнах. Материалы, технология, конструкция, применение: Пер. с чешск.-М.: Мир, 1990-584 с

13. Шарапов В.М., Мусиенко М.П., Шарапова Е.В. Пьезоэлектрические датчики/ Под редакции В.М. Шарапова.-Москва: Техносфера, 2006.-632 с. ISBN 5-94836-100-4

14. Ультразвук. Маленькая энциклопедия. Под редакции И.П. Голямина.-Москва: Советская энциклопедия, 1979. -400 с.

References

1.David L. Churchill. Inertial measurement system with self correction: Patent US 8,010,308 B1 
Int. Cl.: G01C 25/00 (2006.01), G06F 19/00 (2011.01). Aug. 30, 2011, p.20.

2.Компания "ST Software" [Электронный peсурс]. - 2018. - Режим доступа: https://www.stsoftware.nl/index.html.

3. Raspopov V.Ya. Mikromekhanicheskie pribory: Uchebnoe posobie.[ Micromechanical devices: Manuall. M.: Mashinostroenie, 2007, 400p.

4. Matveev V.V. Matematicheskie modeli mikromekhanicheskikh giroskopov LL-tipa [Mathematical models of micromechanical gyroscopes of LL type]//zvestiya TulGU. Tekhnicheskie nauki.2012. Vyp.12, CH1, 205-213 pp.

5. Matveev V.V., Pogorelov M.G. Analiz pogreshnostey mikromekhanicheskikh giroskopov metodom variatsiy Allana [Analysis of errors of micromechanical gyroscopes by method of variations of Allan]//Izvestiya TulGU. Tekhnicheskie nauki, 2015, Vyp.3, 123-135 pp.

6.Petrov P.N. Akustika. Elektroakusticheskie preobrazovateli: Uchebnoe posobie. [Acoustics. Electro-acoustic converters: Manual] SPb.: SPbGUAP, 2003, 80s.

7. Antsev G.V., Bogoslovskiy S. V., Sapozhnikov G. A. Giroskop-akselerometr. [Gyroscope accelerometer]. Patent: RU 2381510, 10.02.2010, 9s.

8. Karimli T.I., Karimli V.I. P'ezoelektricheskiy samonastraivayushiysya giroskop akselerometr [Piezoelectric self-adjusting gyroscope accelerometer]. EA 201500859 A1. 31.10.2016. p.12

9.Pashaev A.M., Karimli T.I., Neymatov V.A. Issledovanie sxemy izmeritel'noy tsepi s p'ezoelektricheskim differentsial'nym akselerometrom [Research of the scheme of a measuring chain with the piezoelectric differential accelerometer]// Vestnik Azerbaydjanskoy Injenernoy Akademii. Tom 9, №2. Baku, 2017, p.7-14.

10. Shabaldin E.D. Metrologiya i elektricheskie izmereniya [Metrology and electric measurements ]. Ucheb. posobie / SHabaldin E.D., Smolin G.K., Utkin V.I., Zarubin A.P.; Pod red. E.D. Shabaldina. Ekaterinburg: Izd-vo GOU VPO «Ros. gos. prof.- ped. un-t», 2006. 282 p.

11. Malinovskiy V.N., Demidova-Panferova R.M. Elektricheskiye izmereniya. [Electric measurements]. Uchebnoye posobiye dlya vuzov. /pod red. Malinovskogo V.N. M: Energoatomizdat, 1985. 416 p.

12. Zelenka I. P'ezoelektricheskie rezonatory na ob'emnykh i poverkhnostnykh akusticheskikh volnakh. Materialy, tekhnologiya, konstruktsiya, primenenie: Per. s cheshsk. [ Piezoelectric resonators on volume and superficial acoustic waves. Materials, technology, design, application: Translation. from Czech ]-M.: Mir, 1990-584 s.

13. Sharapov V.M., Musienko M.P., Sharapova E.V. P'ezoelektricheckie datchik [Piezoelectric sensors] / Pod pedaktsii V.M. Sharapova.-Moskva: Tekhnosfera, 2006.-632 c. ISBN 5-94836-100-4
14. Ul'trazvuk.
Malen'kaya
entsi-

klopediya.[Small encyclopedia]. Pod redaktsii I.P. Golyamina.-Moskva: Sovetskaya entsiklopediya, 1979. $400 \mathrm{~s}$. 